\title{
Theorizing the Experience of Travel in the Film North 24 Kaatham
}

\author{
Anupama A. $\mathrm{P}^{1}$ \& Vinod Balakrishnan ${ }^{2}$ \\ ${ }^{1}$ Research Scholar, Department of Humanities and Social Sciences. National Institute of \\ Technology,Tiruchirappalli.Tamil Nadu. India.E-mail: anupriya2621@gmail.com \\ ${ }^{2}$ Professor, Department of Humanities and Social Sciences. National Institute of \\ Technology, Tiruchirappalli. Tamil Nadu. India. E-mail: winokrish@yahoo.co.uk
}

\begin{abstract}
There are two spaces in Anil Radhakrishnan's travel narrative, North 24 Kaatham (2013), the topographical space outside and the psychological space inside. The film is read as a dialectical tension that plays out in the character of Harikrishnan who suffers from an obsessive compulsive disorder. The fateful journey of Harikrishnan on a day of harthal (general strike) is, to all appearance, topographical though it is, in equal measure, a psychological one. The paper, through a formalist analysis of the film, draws a correspondence between the two journeys of Harikrishnan in the company of fellow passengers: Gopalan and Narayani (Nani), in order to demonstrate Hari's transformation from a self-absorbed individual towards a sociable human being. The argument is structured by combining Walter Benjamin's idea of "aura" and Gaston Bachelard's dialectics of space to explain the protagonist's psycho-spatial transformation.
\end{abstract}

Keywords: travel, outside/inside, topographical space, formalist analysis, spectator experience

\section{Introduction}

Films affectively shape the spectator's notions of the social world in which he or she is situated. One of the most notable cinematic tropes can be found in the genre of travel films where the visualization of a life-changing experience can extend even to the spectator who, identifying with the protagonist, embarks on the journey though at the virtual level. Through a visual analysis of the film North 24 Kaatham (2013), the paper brings out the cultural implications and the impact of travel that have been astutely suggested by the debutante director, Anil Radhakrishanan.

While the topographical Kerala space in the film unravels in the journey from Trivandrum in the south to Kozhikode in the north, the film can also be conceptualized through Walter Benjamin's concern for the work of art in the age of mechanical reproduction where human perception changes with the "aura" of the work of art. Benjamin explains "aura" as, essentially, the sense of the spatial set off by a representation; as "the unique phenomenon of distance, however closer it may be" (1969, p. 219). While that reference may have been made with respect to mechanical reproduction, one may beneficially appropriate the concept to characterize film art that represents experience that is uniquely human. In a travel narrative, one is looking at dynamically shifting locations which present themselves as a cascade of situations calling for responses that are not predictable. The participating spectator who connects with the places, cultures, and the distances covered by the protagonist correspondingly and constantly establishes a new mental space. Thus, by utilizing Benjamin, this paper explores how the protagonist's

(C) AesthetixMS 2020. This Open Access article is published under a Creative Commons Attribution Non-Commercial 4.o International License (http://creativecommons.org/licenses/by-nc/4.o/), which permits non-commercial re-use, distribution, and reproduction in any medium, provided the original work is properly cited. For citation use the DOI. For commercial re-use, please contact editor@rupkatha.com. 
journey from the South to the North of Kerala can be experienced as a mental reproduction of a unique human experience by the spectator. This reproduction is studied through the dialectics of outside and inside that exemplifies the relation between the physical and mental spaces.

By adopting a formalistic analysis of the film, the paper takes cognizance of the different shots and angles and then assembles them into coherent scenes, allowing the spectator to experience the social contexts of Kerala in a whole new light as a result. This approach has been used to study the character development of Hari through the narration of plot in order to convey the stress and obsession he undergoes during the journey from Paravur to Kozhikode. Such an approach examines each and every incident that take place inside and outside of Hari as significantly demonstrating the function of the travel genre where one kind of space, say, the topographical, reconfigures another kind of space, say, the psychological.

The film narrates the expedition of three strangers on the day of a harthal (general strike) covering 24 kaathams, that is, 384 kilometers. In Malayalam, Kaatham refers to a unit of distance equal to 16 kilometers. Director Anil Radhakrishnan picturises the journey as a cascade of amusing events that takes place in the lives of Hari (Fahad Fassil), Gopalan (Nedumudi Venu), and Narayani (Swati). The spectator, who is situated in the topographical space captured by the film, responds to the series of amusing episodes in the text in a culturally-mediated way. The spectator's engagement with the episodes brings together the geographical and sociological landscapes as they involve places and people, thereby, necessary for the spectator's understanding of the film. That understanding of the spectator is connected as much with the generic significance of the travel narrative as much as it is connected with what Alu and Patrica Hills define as the idea of gazing or seeing. According to them, the gaze is what transfers the text, whether in written or in visual form. They state that "gazes express a narrative space from which narrator and reader scrutinise, judge and categorise the varied cultures and societies they explore through writing and reading" (2018, p.1). The gaze gives the spectator a travel experience along with the specular position to scrutinize and judge the socio-cultural space of Kerala. Besides, one evaluates the spectator's urge to participate in the narrative because he/she is less-harassed in this vicarious journey compared to the distressing one undertaken by the protagonist. Amy Lynn Corbin in her article "Travelling through cinema space: the film spectator as tourist" argues that "part of the pleasure of spectatorship is imagining one is inhabiting a virtual space, distinct from the real space of viewing" (2014, p. 314).

This paper presents the spectator's relationship with the film as, essentially, a spatial one. Hence, the kaatham in the title has cultural significance, as it lends specificity to the Kerala spectator's idea of distance. That the spectator relates to the distance covered in the journey in native terms brings into relief the basic and insightful argument about spatiality by Michel de Certeau who asserts that '[s]patial practices in fact secretly structure the determining conditions of social life' (de Certeau, 1984, p. 96). This paper builds on the culture-specific spatiality in order to explore the complex relationship between the physical and the mental spaces within the spectator. The vicarious journey of the spectator, thus examined, convinces one that a physical space can significantly influence the spectator's mental space in order to re-configure patterns of socialization. Harikrishnan, the protagonist in the film, is that specular site where the topographical and the psychological come together to play out their dialectics. 


\section{The Inside Space: Harikrishnan's Obsessive Compulsive Disorder}

Harikrishnan, a techie, is an introvert and rude to his family and colleagues owing to his obsessive-compulsive traits. But he is liked by his boss simply because he produces brilliant work. It is a relationship in which the workaholic is accommodated for being productive than for possessing amiability for true friendship. The establishment shots drive home the fact that Hari is averse to travelling and, in fact, to anything which disturbs his precious routine. He wakes up from bed turning in a particular direction and moves around his room. He is particularly finicky about the way he arranges his bed linen, ensuring that it is totally free of wrinkles. Likewise, he follows an unchanging pattern in the way he brushes his teeth, as well as how he uses mouth wash and mouth freshener. He uses two pairs of slippers -- one he wears in the washroom and the other is used for going outdoors. He follows this pattern regularly and never deviates from it. He is also obsessive with cleaning his wash basin and toilet seat. He repeats this ritual of cleaning regularly and even makes strange sounds while doing his exercises. He brings his own plate for breakfast and ensures the same fixed quantity of food is to be consumed daily. Needless to say, he never allows anyone to clean his room for fear that such will disrupt his daily activities.

Though efficient at technical tasks, his behaviour presents considerable social challenges. His colleagues find it difficult to cope with his emotional outbursts and strange mannerisms. He often fights with his colleagues even when someone accidentally sits on his chair. He has his own particular time to reach the office and makes it a point to climb the stairs and never uses elevators. He does not touch the handles of doors and taps with bare hands, and waits for someone to open the door if he finds himself stuck inside. He lacks communication and interpersonal skills which cause him to circumvent stressful situations by simply avoiding those which are likely to disrupt his routine. However, his commitment to these routines is upset and he is transformed completely after his meeting with two strangers, Gopalan and Nani during a train journey. The latter become the proverbial mirror on which the bizarre and idiosyncratic mannerisms of the protagonist play out; their reactions serve as an expressionistic outing of the personality of Hari for the benefit of the spectator.

\section{Transformation of Harikrishnan: The Outsider}

The travel genre has been a trusted vehicle in fairy tales, mythological stories, scripture, and fictional narratives as it ties up change of human nature with displacement or change of topos. Reflecting on the preparation of Che Guevara for the life of a revolutionary, Sofia Sampaio calls his motorcycle journey across the Latin American continent which has been captured on film as a 'life-changing experience'. She analyses that "travelling is a source of transformation, sidestepping more banal kinds of experiences." (Sampaio, 2014, p.184). Radhakrishnan's film depicts how the travel experience leads the protagonist to unexpectedly transform into a sensitive and caring individual despite the difficulty of overcoming his private demons.

While travelling from the South of Kerala, from Paravur near Kollam to Kozhikode in the North, Hari finds himself haplessly stranded along with Gopalan and Narayani on a harthal day. The various incidents in the film help him to move beyond his obsessive traits by leaving him no other option than to transform as a normal person. In the process, Hari the insider turns into Hari the outsider under the impact of the social relations which compel him into interactive situations with others. For him, the journey becomes a learning experience. When Nani and Gopalan enter a toddy shop and order tapioca and fish curry, Hari is revolted to find the place terribly untidy and 
refused to have food from there. But when he is compelled to use unclean toilets in bus stations, to travel as a pillion rider on bikes, to be taken in a shabby police jeep, and later in a minister's car, he gradually manages to overcome his obsessive compulsions by realising the value of human bonding, helping others, and forming relationships.

The toddy shop, tapioca and fish curry are clear cultural markers. The fish curry is as much a geographical marker as much as it is a culinary marker. Kerala is a coastal state with a history of bringing waves of invaders like the Arabs, the Portuguese, the French, the British who were drawn to her for her spices, especially, pepper. A spicy fish curry is an identity tag of Kerala where the fish curry changes in colour, consistency, condiments, texture and taste as one moves geographically from the South to the North. Like the road that binds one place to another, the geographical particulars of Kerala are connected by the toddy shop and tapioca. The toddy shop, with the distinctive white board and bold black letters, dots the length and breadth of the state. While Kerala is a rice producing state, it is culturally identified as the land of tapioca which is the staple diet. And one goes to a toddy shop not to have rice but tapioca and fish. Gopalan and Nani comfortably ease into the ambience of a toddy shop which is naadan or typically of the countryside. Hari's refusal to take food from the unclean plate suggests his reluctance to integrate into the cultural space which he has been treating as the hostile other space.

Later in the narrative, Hari finds himself in a situation where he is pushed to understand the meaning of loss when news reaches them that Gopalan's wife has passed away. After returning from Gopalan's house Nani asks Hari "Shall we go and eat something?" As a sign of his transformation he had food with her and licks his finger the way Nani does. Hari, for the first time in his life, enjoys the taste of having food with hands. The fork and spoon always came between him and his taste of food. By licking his fingers, Hari signals the willingness to experience more of human contact without the mediation of lifeless cutlery. This contact with the fingers must be seen as a synecdoche of his change. From the part (finger) he graduates to the whole which happens when he begins to see Nani as more than a fellow passenger.

This instance explains Hari's transformation from OCD to a normal person.He even begins to love Nani, which develops in him the value of bonding and a sense of man-woman relationship in life. The film ends with Hari telling his doctor that he feels a sense of loss after the trip. That sense of loss is proof of his changed attitude to life. It implies that he has become able to offload the baggage of obsessions that denied him a social acceptance. It is, as if, the journey itself happened in the brain of Hari where he finds himself moving from a spot of selfishness in the orbito frontal lobe (corresponding to the beginning of the adventure in Paravur) to the spot in the hypothalamus (corresponding to the terminus of the adventure in Kozhikode) which, neuroscientifically, through the secretion of Serotonin, restores the miscommunication between the frontal lobe and the deeper communication spots.

The following figure demonstrates how Hari transforms under the impact of the social spaces in which he is situated. On one level, there is a shift in brain activity which takes place as Hari's social contacts with Nani and Gopalan are developed. The physical space which he occupies is the place where the spectator watches him as a person with obsessive compulsive disorder. During his journey from the South to the North of Kerala, however, this space becomes a shared social space rather than an individualized one when Nani and Gopalan help him to develop his mental emotions outside of the closed space in which his own thought process is clearly restricted. For example, when Hari learns that Gopalan's wife has passed away, he, for the first time in life, encounters a state of mind which breaks the registered patterns of routine which he 
previously constricted his thinking. This new mental emotion later transforms itself into a strong mental activity which leads him to find the courage to do things he had avoided before, such as eating from ordinary shops, walking barefoot, touching a new-born baby, and travelling in bikes and police jeeps.

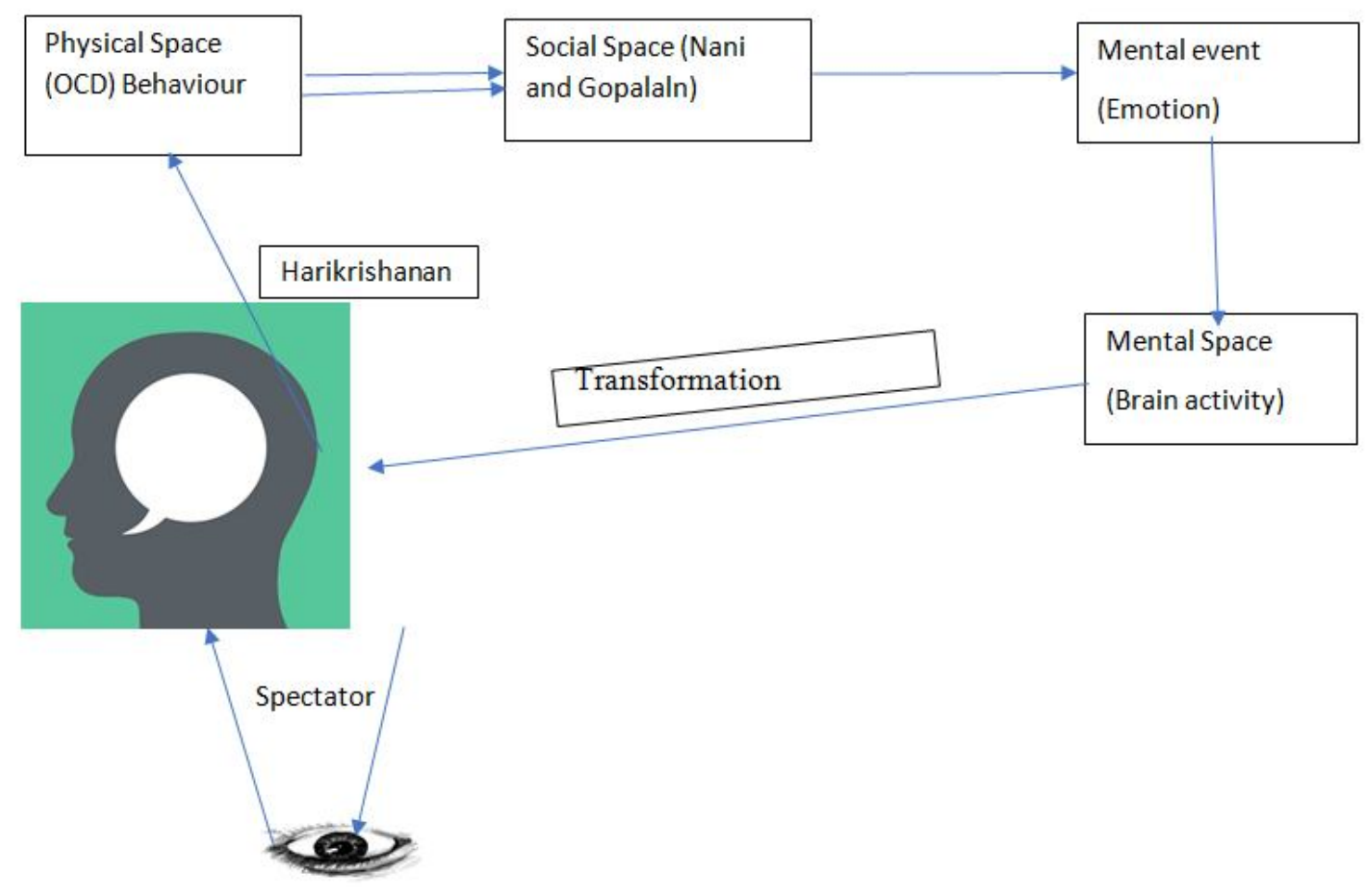

Fig. 1: The flow of mental transformation of Spectator

Like Hari's colleagues, his parents too are bewildered at his change in behaviour. They express their anxiety with Hari's doctor:

Doctor: Maya what is your problem now? Is it because Hari no longer laughs in the morning?

Maya: He has started to take two more idlies.

Doctor: He must be having a better appetite now.

Siddharth (Hari's brother): Doctor, he has gone mad. Something has gone wrong with him. He used to walk to and fro but now he does not. It is after that trip he went on. Doctor, what do you think is the reason?

Doctor: It could be self-realization.

Hari's transformation is, however, not limited to his own private mental space. The film allows his personal transformation to be recreated by the spectator who finds himself or herself immersed in these places, cultures, and distances, thereby, enabling them to establish a new mental space of his/her own in the bargain. 


\section{The Dialectics of Outside/Inside: Mental Space/Physical Space}

Dialectics is an argumentative method set out to establish the reason or truth behind a fact. One cannot conceive the idea of a journey without some idea of spaces where one limits one's attention to the outside. The cinematic experience persuades one to see that it is also important to consider the problem of "inside spaces." There are times, for example, when life is seen through a narrower spectrum, which closes one's mental space and causes one to form delusive notions. Silvana Dunat provides a useful explanation for this in her article "Film space as mental space," in which she argues that film-space must be explored as a mental space rather than as a purely physical one. Cinema causes a mental space to be actively constructed based on one's perception of the images projected onto the screen. At the same time, it is also important to note that this perception never occurs in a vacuum. It can only be constructed with reference to the broader contexts of one's experience of the world and of the society at large. Dunat explains that these spaces must be conceptualized as real, virtual, and mental which is necessary for the construction of meaning.

With respect to the dialectical tension which a spectator undergoes, it may be posited that $\mathrm{s}$ /he encounters the physical and social space for the construction of meaning. On one hand, such a space is the creative outcome of the spectator's encounter with the physical space. However, the spectator also sees that Hari's travel is not only a displacement of physical distances within Kerala but, equally, a journey to transform himself mentally. One may consider Mikkonen's thesis that adds a temporal dimension to Sampaio's spatial thesis; that travel actually has a much broader definition than the stereotypical concept of traversing physical distances. In fact, he defines it as "any series of events" which "humanizes' the experience of time and space." In North 24 Kaatham, for example, the travel-experience in the film is a projection of the experience of Kerala society as it moves through a given space within a given time (on a harthal day).

Kerala society is the site of the conceptual fusion of both the outside and inside spaces for Hari. The change that the spectator is able to see in Hari even as the journey unfolds from stationto-station is indicated by the protagonist's own capacity to see which graduates from merely seeing himself to registering the social presence of others to whom he is bound. Bernard McGrane refutes stereotypical notions that travelling is only defined by physical movements, when he argues that "to travel is to see-travel is essentially a way of seeing, a mode of seeing: it is grounded in the eye, in our visual capacity" (1989, p. 116). In this film, the significance of the journey is in its exposition of Hari's transformation caused by his newly acquired capability to see the reality of social existence for himself. That capability to see or see into himself is a displacement on a personal level rather than merely in his physical location. As a result of travelling, Hari sees the world around him in new ways, as his gaze into the outer world presents a narrative space (physical space) for the spectator. The spectator, on his or her part, then, scrutinizes the social space by forming a connection with the protagonist and the social relationships he acquires to "conjure up the appearances of something that was absent" (Berger, 1972, p. 10).

Gaston Bachelard, in his essay "The Dialectics of Outside and Inside" explains, for example, how the house and domestic space must be understood through people's intimacy to their home which cannot be reduced to an analysis of physical location alone. This approach provides a strong theoretical framework to understand the spatial relations a person inhabits. It implies that the house is a domestic space of memories and moments of human life rather than a neutral container of geometrical coordinates. In the film, one can see that Hari's domestic space is 
not only his room but also his beloved routine activities. He becomes so comfortable with them, in fact, that he ends up hating anything that might threaten to break his precious routine. Bachelard's theorization of domestic space suggests that Hari's comfort zone is not only a place but a set of memories and moments of life as well.

Bachelard calls the outside and the inside as "a dialectic division" which shapes and restricts our experience of the space. The dialectics of Hari as insider and Hari as outsider open up two possibilities for the spectator. On the one hand, it allows the spectator to closely scrutinize their subjective experience. In addition, it helps them to come to terms with their complex thoughts and emotions. Bachelard claims:

Outside and inside are both intimate-they are always ready to be reversed, to exchange their hostility. If there exists a border-line surface between such an inside and outside, this surface is painful on both sides...Intimate space loses its clarity, while exterior space loses its void, void being the raw material of possibility of being. (1958, p. 218)

In other words, Hari's dialectical tension between the outside (transformed) and the inside (OCD) creates the same dialectical tension in the spectator at the point Hari's inside space affects the spectator. The spectator is led to the position of considering whether one should be excessively obsessed with the cleanliness. On the other hand, such obsessions will bring out a person's societal relation with other people. Hence, the travelling experience of Hari is equally a cinematic experience for the spectator.

These spatial nuances allow the spectator to uncover, assuming space itself to be language, the notion of 'opening up.' As Bachelard himself defines it: "language bears within itself the dialectics of open and closed. Through meaning it encloses, while through poetic expression, it opens up" (1958, p. 222). This opening up is a way to bring out the complexities in Hari's mind who is an introvert by nature. Hari's open and closed spaces are, in the words of Bachelard, simply "thoughts" (1958, p. 212). They are metaphors that Hari links to every single thing he experiences. Hari first breaks the geometric division when he reaches Kollam station where Gopalan asks Nani, "What is the time?" It is only then that Hari is reminded of his daily routine of going to the wash room. The outside and inside is therefore one of the ways of establishing one's position in the society rather than a matter of individual examination alone. The dialectic is what shapes the space in an active and qualitative manner. For instance, there is a scene in which Hari touches the water barefoot. Similarly, he senses the feel of handling a child for the first time when he takes a new born baby. He is worried whether the child would pee. All these incidents bring out Hari's inside/closed space to the outside.

Hari's social space is, during the journey, taken over by Nani and Gopalan. Along with them Hari experiences the social space of Kerala which leads to his transformation when they have food from a toddy shop, travel in strangers' motorcycles, travel by fishing boat etc. Hari starts to transform when Nani is being kidnapped by thugs who promise to drop them at Ernakulam. During this crisis, the inside space of Hari slowly transforms into Hari the outsider, where he expresses his concern over Nani (rather than only for himself.) Similarly, when Nani gives Mustaka's daughter to Hari, he experiences the intimacy of a new human relationship through the baby. When Hari manages to shed his obsessions, the exterior space is finally freed to enable him to enter into the realm of transformation. The conversation between Nani and Hari proves this shift:

Nani: Are you always like this? 
Hari: How?

Nani: A cleanliness freak.

Hari smiles.

In fact, he breaks into a smile for the first time in his life. At this point, Hari has already created a mental space in the spectator's mind. This scene clearly shows the outside comes out of the inside under the impact of the social space in which he vigorously interacts. This socialization process can only take place in relation with other people. Hari's association with Nani made him feel a sense of loss. Thus he opens up a new door to tell the story of his life for the spectator.

\section{Conclusion}

This paper adopts a formalist analysis to conceptualize the travel film, North 24 Kaatham. It develops the cinematic experience that is shaped by the formal demands of a travel narrative. It traces the dialectical tension a spectator undergoes when s/he travels along with the protagonist Hari whose transformation from a maladjusted individual to a sociable person creates a mental space in the spectator which determines the formation of meaning. A journey provides the physical space to break out of the cramped mental space on account of the tension between the inside and the outside; between the mental and the physical worlds. The film visualizes such a tension and establishes the widening of mental spaces through the physical spaces of socialization.

\section{References}

Alu, G. and Hill, S. P. (2018). "The travelling eye: Reading the visual in travel narratives". Studies in Travel Writing, vol. 22, no. 1, 2018, pp. 1-15. https://doi.org/10.1080/13645145.2018.1470073

Bachelard, G. (1958). “The Dialectics of Outside and Inside.” The Poetics of Space. Translated by Maria Jolas. Boston: Beacon, 1994, pp. 211-231.

Benjamin, W. (1969). “The Work of Art in the Age of Mechanical Reproduction.” Illuminations, edited by H. Arendt. New York: Schocken, 1969, pp. 217-251.

Berger, J.(1972). The Ways of Seeing. British Broadcasting Corp.

Corbin, A. L. (2014). "Travelling through cinema space: The film spectator as tourist." Continuum: Journal of Media \& Cultural Studies. vol. 28, no. 3, 2014. pp. 314 329.https://doi.org/10.1080/10304312.2014.900880

Dunat, S. "Film space as mental space" Semiotica, 2015.207 (2015): 475-487. Retrieved 18 Mar. 2020, from doi:10.1515/sem-2015-0039.

Mikkonen, Kai. “The 'Narrative Is Travel' Metaphor: Between Spatial Sequence and Open Consequence." Narrative, vol. 15, no. 3, 2007, pp. 286-305. JSTOR, www.jstor.org/stable/30219259. Accessed 17 Mar. 2020.

McGrane, B. (1989). Beyond Anthropology: Society and the Other. New York: Columbia University Press.

Sampaio, Sofia. "Watching narratives of travel-as-transformation in The Beach and The Motorcycle Diaries". Journal of Tourism and Cultural Change. Vol. 12, no. 2, 2014, pp. 184-199. https://doi.org/10.1080/14766825.2014.915094

North 24 Kaatham. (2013). Directed by Anil Radhakrishnan, performances by Fahad Fasil, Swati Reddy and Nedumudi Venu. E4 Entertainment. 